\title{
Ethical considerations in implementing a biometric co-enrol- ment prevention system in clinical trials in South Africa
}

\author{
J Moodley, ${ }^{1}$ BPharm; S Naidoo, ${ }^{1}$ PhD; N S Morar, ${ }^{1}$ M MedSci; V Govender, ${ }^{1}$ MB BCh; P Charls, ${ }^{2}$ G Ramjee, ${ }^{1,3,4}$ PhD, FRCP \\ ${ }^{1}$ HIV Prevention Research Unit, South African Medical Research Council, Durban, South Africa \\ ${ }^{2}$ Information Technology Services Division, South African Medical Research Council, Cape Town, South Africa \\ ${ }^{3}$ Department of Epidemiology and Population Health, London School of Hygiene and Tropical Medicine, London, UK \\ ${ }^{4}$ Department of Global Health, School of Medicine, University of Washington, USA
}

Corresponding author: J Moodley (Jothi.Moodley@mrc.ac.za)

\begin{abstract}
Preventing co-enrolment in clinical trials ensures participant safety and data integrity. To facilitate co-enrolment checks, a novel biometric coenrolment prevention system (BCEPS) was developed and implemented in 2010 by the HIV Prevention Research Unit (HPRU) in collaboration with the South African Medical Research Council's (SAMRC) Information Technology Services Division. The use of this web-based system to capture participant's identification details in real time was approved by the SAMRC Ethics Committee. BCEPS was implemented at 13 other research organisations conducting clinical trials in South Africa (SA). Participants who screened at the clinical research sites (CRSs) had their names, SA identity or passport number and fingerprints captured onto BCEPS after comprehensive education and discussion. This information was verified at all study visits. If a participant attempted to screen or co-enrol at multiple CRSs, the system flagged this as a potential coenrolment. By addressing the ethical concerns around participant consent and rights, participant confidentiality and privacy, data security and access, and data management and storage, we were able to successfully implement BCEPS within the clinical trials conducted at HPRU, while adhering to the principles of good clinical practice (GCP), including respect for persons, beneficence and justice.
\end{abstract}

SAfr J BL 2016;9:39-41. DOI:10.7196/SAJBL.405

South Africa (SA) has become one of the leading locations for the conduct of clinical trials due to its high-burden of disease, limited access to healthcare, well established research infrastructure and access to suitably qualified investigators. ${ }^{[1]}$ Co-enrolment


or concurrent enrolment of participants into multiple clinical trials
testing therapeutic or prevention agents, is a potential risk in this setting. ${ }^{[2]}$ Co-enrolment has the potential to affect the power of a study that determines the effect of an intervention, bias the study due to possible interactions between the interventions, increase the burden of clinic attendance and study procedures on participants and impact on the safety of the participant due to the possible unexpected interactions between interventions. ${ }^{[3]}$ Reported reasons for coenrolment in this region include access to high quality healthcare at the clinical research site (CRS), study re-imbursements, altruism, access to investigational products such as study gels to enhance sexual pleasure, perceived low risk of being identified by researchers as being co-enrolled, and peer pressure by fellow co-enrollees. ${ }^{[3]}$

In 2008, the HIV Prevention Research Unit (HPRU) of the South African Medical Research Council (SAMRC) identified several participants who co-enrolled in two HIV prevention clinical trials being conducted in close proximity. ${ }^{[2]}$ These co-enrolments fortunately did not impact on participant safety and study outcomes. ${ }^{[4]}$ This incident did, however, raise concerns among sponsors and researchers that co-enrolment in HIV prevention clinical trials is a reality that must be identified and prevented from occurring. ${ }^{[4]}$ When designing any research protocol, it is imperative that investigators consider the potential impact of co-enrolment on study outcomes, safety and burden of study procedures on participants. If co-enrolment has the potential to either augment or negate the therapeutic effect of the interventions or alter the adverse event profile of the intervention being studied, then the possibility of co-enrolment should be avoided. This consideration is especially important when implementing trials in resource-poor settings where trial participants are more likely to attempt to co-enrol. ${ }^{[2]}$

\section{Biometrics co-enrolment prevention system}

The biometrics co-enrolment prevention system (BCEPS) is a novel approach used to preventco-enrolment of research participants in multiple clinical trials in SA. ${ }^{[2]}$ This system was developed and implemented by the HPRU in collaboration with the SAMRC Information Technology (IT) Services Division and an external developer in 2010. ${ }^{[2]}$ This is a webbased system, which uses biometric technology to capture participant's personal identification details in real time. ${ }^{[2]}$ In brief, this system is used to capture the participant's full name, SA identity number or passport number (if not South African) and fingerprints. This information is used to determine if a participant has screened and/or enrolled at any other research site that is listed in the co-enrolment database. The system can also link studies which are pre-determined by the investigators, to allow co-enrolment.

BCEPS is currently utilised by 26 CRSs within 13 research organisations in SA, and to date has more than 26500 participants registered on the system. All participating research organisations sign a memorandum of agreement (MOA) which is a legal agreement between the research organisation and SAMRC. On signing the MOA, the participating research organisations agree to abide by the terms and conditions applicable to the use of the 
system. Prior to its implementation in 2010, BCEPS was externally evaluated by an independent company to ensure its functionality. More recently the system was audited by a study sponsor to verify compliance with applicable regulations and guidelines for the use of computerised systems in clinical trials and for compliance with the MOA.

\section{Regulatory oversight}

Research involving humans must be scientifically sound and conducted in accordance with basic ethical principles as outlined in the Declaration of Helsinki and by the Council for International Organisations of Medical Sciences (CIOMS): respect for persons, beneficence, and justice. ${ }^{[5,6]}$ Furthermore, SA has stringent ethical guidelines stipulated by the National Health Act (2003) and the SA Good Clinical Practice (GCP) guidelines which ensure that the rights, wellbeing and safety of individuals participating in clinical trials are protected. ${ }^{[7,8]}$ In addition, clinical research trials must be approved by the local ethics committee Medicines Control Council (MCC) and be registered with the South African National Health Research Ethic Committee (NHREC) trial registry. Registration of clinical trials on NHREC ensures that all SA researchers are aware of the current clinical trials being conducted.

BCEPS was initially approved by the SAMRC ethics committee in 2010 for use in trials conducted within the HPRU and reviewed again in 2014. During the 2014 review process, the ethics committee commented that 'the fingerprint system is good to have, but it should be balanced with ethics principles'. The following considerations were raised by the committee:

- If the fingerprint database will be kept indefinitely, please provide more and detailed information on the safety and security measures that will be taken to preserve confidentiality. Who will be accountable for this sensitive information? The committee asked for guidelines and standard operating procedures (SOPs) for the use of fingerprints to give them assurance.

- Separate consent should be added to the informed consent where the participant agrees to give their fingerprints to other researchers.

- If a participant refuses to give their fingerprints, will they be excluded from the study? Can a participant ask to withdraw their fingerprint data from the database?

Based on the above mentioned findings, the following ethical considerations have been implemented:

\section{Participant consent and rights}

To achieve understanding of BCEPS, participants are required to read and sign a co-enrolment participant information sheet (PIS), approved by the SAMRC ethics committee. This PIS includes comprehensive information on the purpose and use of BCEPS. Participants also receive detailed counselling regarding the risks of co-enrolment at their screening, enrolment and at follow up clinic visits. The CRS staff must ensure that while consent is required to add the participant's data to BCEPS, the participant does have a right to refuse, or to withdraw from BCEPS at any time. Investigator discretion is used to decide if it is safe for the participant to enrol in the study; or continue with study product use without co-enrolment checks if the participant refuses. To date, at HPRU, no participant has refused consent or withdrawn from BCEPS.

\section{Participant confidentiality and privacy}

When implementing BCEPS, care is taken to ensure the participants right to privacy and confidentiality is maintained as the system is used by many CRSs. The system is managed externally by the SAMRC IT Services Division, in order to preserve the confidentiality and security of participant data. This process is explained to participants in the waiting room. All SAMRC IT staff sign a confidentiality agreement. All correspondence regarding BCEPS queries is done via email between the participating CRSs and the SAMRC IT Services Division. It is therefore crucial that this communication does not compromise the confidentiality of the participants. Delegated CRS staff, trained to send queries to a dedicated email address, ensure that only essential participant information such as the name and SA identity number are shared. There is no link to the unique study identity number and any participant-specific study data. A copy of this correspondence is filed at the CRS and the email is deleted to ensure the protection of participant confidentiality.

\section{Data security and access}

To ensure that the system is secure and to prevent unauthorised access to the data, BCEPS is password protected and has two levels of authentication as a security measure. To gain access to the system authorised and trained study staff are required to login to a Secure Socket Layer Virtual Private Network (SSL VPN) portal using a username and password and thereafter login again using their fingerprint in order to access the application. The SSL VPN creates an encrypted connection between the user's computer and the server. The BCEPS server hosting BCEPS is protected by a CISCO Systems Inc. firewall located in a demilitarised zone (DMZ). Fingerprints are stored as an encoded text string and not as an image and therefore cannot be copied or reverse engineered. No data, such as study identifiers or participant clinical data are stored locally, or on computers. When a participant is in attendance for a study visit at the CRS, their fingerprint is scanned to check if they are currently enrolled on the system. The system alerts the user if a co-enrolment is detected in another study or at another site. The researcher then has the option to contact the investigator from the other site and/or study to discuss the participant's current status in the study in order to assess if it is safe to proceed with study participation. This process contributes to participant safety and data integrity.

\section{Data management and storage}

Trained SAMRC IT staff members install BCEPS at each participating research site. They ensure that the delegated CRS staff are trained on the system and provided with a data entry guide that contains instructions on the use of BCEPS. Research staff then follow the site-specific standard operating procedures (SOPs) on the prevention of participant co-enrolment.

Staff members who experience technical problems with BCEPS are encouraged to contact the SAMRC IT Services Division that subsequently resolves all system related issues in a timeous manner. Data for each participant are maintained on the system for up to 15 years but can be removed by the SAMRC IT if requested by the participant. The data are backed up daily on the server and are also duplicated on the SAMRC disaster recovery site every night. Should internet connectivity be lost at site, there is an option to connect to the server using a dedicated laptop via $3 \mathrm{G}$. 


\section{Quality assurance}

Quality checks at the CRSs include verifying that the participant's correct name and identity number, as per the SA identity document, has been captured. Daily checks are performed to ensure that no participant has inadvertently been omitted from BCEPS. If a participant presenting at a research site is not captured on the system due to human error, this could have a huge impact on participant safety and data integrity.

\section{Conclusions and recommendations}

Ethically, researchers must protect the rights, safety and wellbeing of participants. It is therefore important for each research organisation to develop a co-enrolment prevention plan or SOP which identifies all potential sources of co-enrolment and implements measures to prevent co-enrolment from occurring. To identify potential sources of co-enrolment, research organisations must engage with each other regularly as well as with all stakeholders and community structures to ensure that there is awareness of all investigational products undergoing clinical trials in the country. More importantly, CRSs within close geographical proximity must maintain open dialogue on their respective research agendas.

Real-time verification of the enrolment status of participants using BCEPS is valuable to prevent co-enrolment. However, its utility is limited if all clinical research organisations conducting clinical trials in SA do not subscribe to one co-enrolment prevention system. By addressing the ethical concerns around participant consent and rights, participant confidentiality and privacy, data security and access, and data management and storage, we were able to successfully implement BCEPS at HPRU. There have been no concerns raised by participants or communities regarding its use in our setting.
Since SA will continue to conduct many large-scale trials, especially in the area of HIV prevention and treatment, it is essential that research organisations engage in robust dialogue on the potential ethical and safety concerns of co-enrolment and implement the use of a single shared database in the country to prevent co-enrolment in multiple studies.

\section{References}

1. Wemos Foundation. The Clinical Trials Industry in South Africa: Ethics, Rules and Realities. Amsterdam: Wemos Foundation, 2013. http://www.wemos.nl/files/ Documenten\%20Informatief/Bestanden\%20voor\%20'Medicijnen'/Clinical Trials_Industry_South_Africa_2013_v3.pdf (accessed 20 June 2014).

2. Harichund C, Haripersad K, Ramjee G. Participant verification: Prevention of co-enrolment in clinical trials in South Africa. S Afr Med J 2013;103(7):491-493. DOI:10.7196/SAMJ.6674

3. Karim QA, Kharsany A, Naidoo K, et al. Co-enrollment in multiple HIV prevention trials: Experiences from the CAPRISA 004 Tenofovir gel trial. J Contem Clin Trials 2011;32(3):333-338. DOI:10.1016/j.ccc.2011.01.005

4. Ramjee G, Coumi N, Dladla-Qwabe N, et al. Experiences in conducting multiple community-based HIV prevention trials among women in KwaZulu-Natal, South Africa. AIDS Res Ther 2010;7:10. DOI:10.1186/1742-6405-7-10

5. World Medical Association. World Medical Association Declaration of Helsinki Ethical Principles for Medical Research Involving Human Subjects. Paris: World Medical Association, 2013. http://www.wma.net/en/30publications/10policies/ b3/ (accessed 20 June 2014)

6. Council for International Organizations of Medical Sciences. International Ethica Guidelines for Biomedical Research Involving Human Subjects. Geneva: Counci for International Organizations of Medical Sciences, 2002. http://www.cioms.ch/ publications/guidelines/guidelines_nov_2002_blurb.html. (accessed 20 June 2014).

7. Republic of South Africa. National Health Act. Pretoria: Government Gazette, 2003. http://www.saflii.org/za/legis/consol_act/nha2003147/ (accessed 20 June 2014).

8. Republic of South Africa, Department of Health. Guidelines for Good Practice in the Conduct of Clinical Trials with Human Participants in South Africa. Pretoria: Department of Health, 2006. http://www.google.co.za/url?sa=t\&rct=j\&q=\&esrc= s\&source=web\&cd=1\&ved=0CBwQFjAA\&url=http\%3A\%2F\%2Fwww.kznhealth. gov.za\%2Fresearch\%2Fguideline2.pdf\&ei=FMO4VMiGCleBPdWygcAK\&usg=AF QjCNFPS2z5fpitrvxT2v5 b4D-5k-BKg (accessed 20 June 2014). 